\title{
Brain damage in fatal non-missile head injury
}

\author{
J HUME ADAMS, ${ }^{*}$ DI GRAHAM,* GRACE SCOTT, ${ }^{*}$ LILIAN S PARKER, $\dagger$ \\ AND D DOYLE*
}

From the University Departments of *Neuropathology and $\dagger$ Neurosurgery, Institute of Neurological Sciences, Southern General Hospital, Glasgow G51 4TF, UK

The most important factor governing the outcome in a patient who sustains a non-missile head injury is the damage sustained by the brain. Some of this may occur at the moment of impact and may be so severe that the patient never recovers consciousness; but in many patients who experience a lucid interval after their original injury and then become unconscious it is clear that the principal brain damage has not occurred at the moment of impact. This is because the original injury-sometimes apparently of no great severity-has initiated a dynamic process that has led to further, or secondary, potentially fatal brain damage.

There are therefore two principal reasons for undertaking comprehensive neuropathological studies on patients who die as a result of a head injury. The first of these is to define as precisely as possible the sequence of events that has led to the fatal outcome. It is only by this means that one can separate immediate impact injury, which by definition is not amenable to treatment no matter how good the medical services are, from brain damage brought about by a complication of the original injury, and at least some of which might have been preventable had the complication been recognised earlier. The second reason is that it is only by defining the wide spectrum of brain damage that may be present in a fatal head injury that one can gain some insight into the types of damage that may be present in patients who remain permanently brain-damaged after a head injury. Certainly one of the principal objectives of the present study was to establish as close clinicopathological correlations as possible in the hope that more comprehensive neuropathological information might to some extent contribute to patient management.

Numerous valuable studies have been published in the course of the past 20 years ${ }^{1-7}$ on the types of brain damage that are likely to be encountered in patients who have died as a result of a non-missile head injury. They are, however, open to certain

Received for publication 6 May 1980 criticism since they fall into two distinct and rather disparate groups: the first is composed of large numbers of cases subjected only to macroscopic assessment of the brain when inevitably much of the brain damage remains unrecognised, while the second consists of small series, albeit subjected to a detailed neurohistological study, but too selective clinically to be of much general value.

The reasons for the paucity of comprehensive neurohistological studies are not hard to find since a full histological analysis of a brain requires the examination of many large sections, preferably embedded in celloidin. This is a time-consuming exercise which requires considerable specialist expertise on the part of both the technician and the pathologist. There has therefore inevitably been a tendency, often contributed to by forensic pressures, for pathologists to lay particular stress on the readily identifiable macroscopic abnormalities such as fracture of the skull, intracranial haematoma, and cerebral contusions. Indeed, only too often is death ascribed to fracture of the skull and cerebral contusions when neither of these may even have contributed to the patient's loss of consciousness! We, however, have become increasingly aware of the more subtle types of brain damage that are almost impossible to identify macroscopically if the brain is sliced cursorily in the postmortem room and that can account for severe cerebral dysfunction and death in head injured patients. Yet a better understanding of these not uncommon types of brain damage would be of considerable help to the general pathologist and to the forensic pathologist faced with an almost normal looking brain in a patient known to have been unconscious for varying periods of time after a head injury.

The brief account of brain damage due to a nonmissile head injury that follows is based on a comprehensive neuropathological study ${ }^{8-12}$ of a consecutive series of fatal cases, and, as a result of these studies, our current approach to the pathology of brain damage in non-missile head injury is as follows: 
Immediate impact injury

Contusions and lacerations

Diffuse damage to white matter

Other types of diffuse brain injury

Primary complications

Intracranial haemorrhage

Brain swelling

Secondary complications

Brain damage secondary to raised intracranial pressure

Hypoxic brain damage

Infection

\section{Material and methods}

During the five-year period 1968-72, 151 necropsies were undertaken on patients with head injuries who had been managed by the Department of Neurosurgery in the Institute. Since all of the patients had survived their injury long enough to be referred to a neurosurgical unit, there are very few patients in the series who survived less than 12 hours. There were 123 males and 28 females; the age range was 8 months to 86 years; and the duration of survival ranged from 6 hours to 21 months (Figs 1 and 2). The majority of the injuries were attributable to road traffic accidents $(46 \%)$ or to falls $(43 \%)$. Blows to the head accounted for $9 \%$, while the remaining $2 \%$ were crush injuries.

The clinical records were assessed independently by the Department of Neurosurgery with particular reference to any deterioration in the conscious level after a lucid interval, defined as whether or not the patient had talked after the injury. ${ }^{13}$ If the patient had talked but had not been completely rational, the lucid interval was defined as being partial. As a result of this study it was established that $58(38 \%)$ of the 151 cases had experienced a complete (25; $16 \%)$ or partial $(33 ; 22 \%)$ lucid interval after injury.

The brains and spinal cords were fixed in $10 \%$

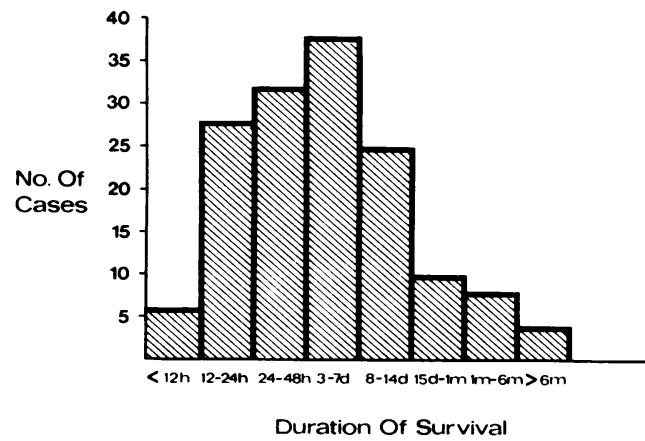

Fig. 1 Duration of survival of the 151 cases (from Adams et al. ${ }^{10}$ ).

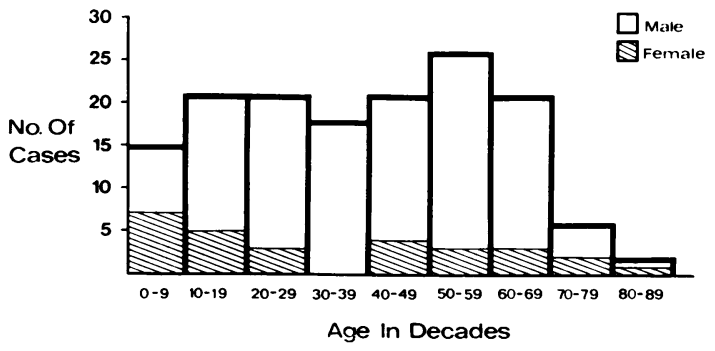

Fig. 2 Age and sex of the 151 cases (from Adams et al. $^{10}$ ).

formol saline for at least three weeks before dissection. After transection of the mid-brain, the cerebral hemispheres were cut in the coronal plane into slices $1.0 \mathrm{~cm}$ thick. Large representative blocks of the cerebral hemispheres and the cerebellum were embedded in celloidin, and $30 \mu \mathrm{m}$ thick sections were stained by Nissl's method with cresyl violet and by Woelke's modification of Heidenhain's technique for myelin. The brain stem was cut into seven to nine blocks. One block each of mid-brain, pons, and medulla were embedded in celloidin, and $30 \mu \mathrm{m}$ thick sections were stained by the Nissl and Woelke methods. Frozen sections were cut from the remaining blocks and stained with oil red $\mathrm{O}$ and by the Marchi technique to demonstrate the breakdown products of myelin. These blocks were then embedded in paraffin wax, and sections were stained with haemalum and eosin, by Luxol fast blue/cresyl violet, by the Glees or Palmgren techniques for axons, and by the Naumenko and Feigin technique for microglia.

The sections were examined with a Watson zoom stereo-microscope with transmitted light at a magnification between $\times 10$ and $\times 50$. Much of the analysis could be done at these magnifications, particularly in the longer surviving patients when it was easy to recognise loss of neurons and reactive changes. In shorter surviving patients, however, where there were foci suggestive of early neuronal necrosis, the lesions were accepted as genuine only if the specific cytological abnormalities of ischaemic nerve cell change with or without encrustations $\mathbf{1 4 - 1 6}^{16}$ could be identified by conventional light microscopy. It was also possible in each case to assess whether or not the intracranial pressure had been high during life on the basis of the histological criteria we have already defined. ${ }^{17}$

All macroscopic and histological abnormalities identified in the brains were charted on a series of line diagrams of the cerebral hemispheres, the cerebellum, and the brain stem, and these diagrams formed the basis of the present analysis. 


\section{Results}

FRACTURE OF THE SKULL

There was a fracture of the skull in 120 of the 151 cases $(79 \%)$; thus about one in five of the patients in this series who died as a result of a non-missile head injury did not have a fracture of the skull. Of the 120 fractures, 69 were centred on the mid-line of the calvaria (55) or were restricted to the base of the skull (14) and therefore gave no indication as to the site of injury. In 51 patients the fracture was restricted to one side of the calvaria or was predominantly unilateral, and in $\mathbf{3 0}$ of these there was a corresponding laceration or bruising of the scalp. It seemed reasonable to assume that in these 30 patients the site of the fracture was a fairly accurate indication of the site of the injury, and that from these cases one could gain some indication as to the

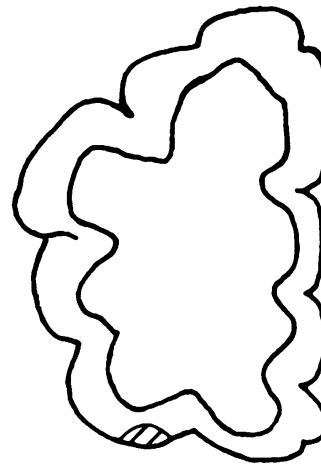

L. Frontal

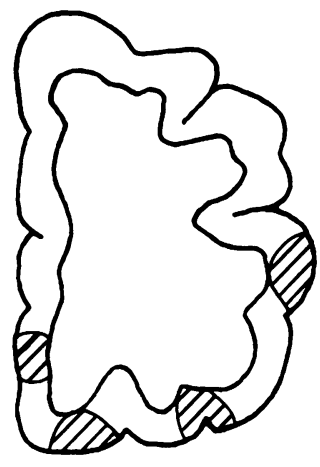

R. Frontal
Fig. 3

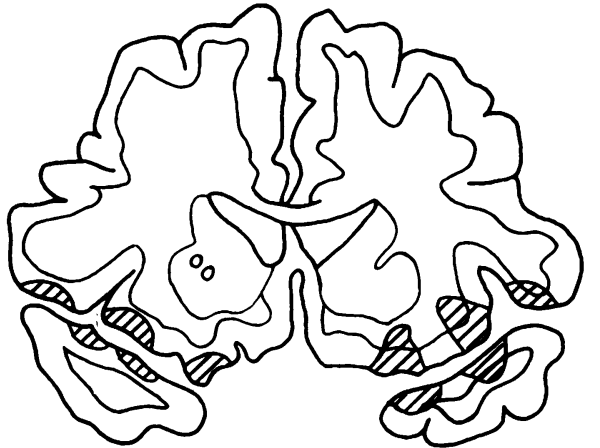

L. Sylvian Fissure $C I=1 \times 3=3$

Fig. 4 distribution of brain damage in relation to the site of injury.

\section{IMMEDIATE IMPACT INJURY}

\section{Cerebral contusions and lacerations}

These are the hallmark of a head injury and occur as haemorrhagic lesions, particularly at the crests of gyri. In an attempt to assess contusions more precisely, we have developed a contusion index (Figs 3, 4 and 5) which has allowed the depth and extent of contusions in various regions of the brain to be expressed quantitatively. ${ }^{12}$ The regions chosen-the anatomical locators-were the frontal, temporal, parietal, and occipital lobes, the cortex above and below the Sylvian fissures, and the cerebellum. Contusions in each anatomical locator are graded according to depth -0 (absent) to 3 (extending into

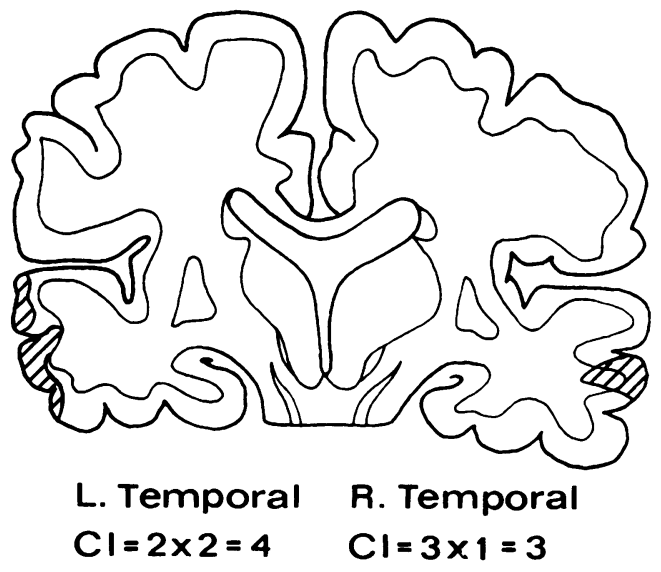

Fig. 5

Figs 3, 4, and 5 Three representative line diagrams of the cerebral hemispheres to show how the contusion index $(\mathrm{CI})$ is derived for six of the anatomical locators re,ferred to in the text (ie, left and right frontal lobes, cortex adjacent to left and right Sylvian fissures, and left and right temporal lobes). The CI for each locator is obtained by multiplying depth (the first digit) by extent (the second digit $)$ and can range from $0(0 \times 0)$ to $9(3 \times 3)($ from Adams et al. ${ }^{12}$ ). 
the white matter)-and extent- 0 (absent) to 3 (very extensive). The contusion index (CI) for each anatomical locator is derived by multiplying depth by extent and can therefore range from 0 (contusions absent) to 9 (deep and extensive contusions). The total contusion index (TCI) for a given brain is obtained by adding the CIs of all the locators, left and right, and can range from 0 to 108. Mean contusion indices (MCI) for specific anatomical locators and mean total contusion indices (MTCI) can be derived for the whole group or for subsets.

An analysis of cerebral contusions in the present series $^{12}$ showed that the MTCI (whole brain) was 16.6. In seven of the 151 fatal head injuries the MTCI was 0 , while in 13 patients contusions were minimal, that is, a CI not more than 1 in any of the locators. The 15 patients (ie, $10 \%$ of the series) with the highest MTCIs were classified as having severe contusions (all had a MTCI of > 32). The MCIs for the various anatomical locators are given in Table 1, the distribution of contusions in the 30

Table 1 Distribution of contusions in entire series $\left(n=151:\right.$ from Adams et al. $\left.{ }^{12}\right)$

\begin{tabular}{|c|c|c|}
\hline \multicolumn{2}{|l|}{ Locator } & \multirow{2}{*}{$\frac{M C I}{5.7}$} \\
\hline Frontal lobe & & \\
\hline Temporal lobe & & $5 \cdot 4$ \\
\hline Sylvian fissure & & $2 \cdot 7$ \\
\hline Occipital lobe & & $1 \cdot 2$ \\
\hline Parietal lobe & & 0.7 \\
\hline Cerebellum & & 0.9 \\
\hline MCI for cerebral hemispheres & $15 \cdot 7$ & \\
\hline MTCI (whole brain) & $16 \cdot 6$ & \\
\hline
\end{tabular}

Table 2

(a) Contusions in patients with localising fractures $\left(n=30:\right.$ from Adams et al. $\left.{ }^{12}\right)$

\begin{tabular}{llllll}
\hline & \multicolumn{2}{l}{ MCI } & & \\
\cline { 2 - 3 } & \multicolumn{2}{l}{ Fracture on left $(n=16)$} & & Fracture on right $(n=14)$ \\
\cline { 2 - 3 } & Left & Right & & Left & Right \\
\hline Frontal lobe & 2.9 & 2.5 & & 3.4 & 3.6 \\
Temporal lobe & 3.3 & 2.2 & & 4.1 & 4.1 \\
Sylvian fissure & 1.0 & 1.7 & & 1.1 & 2.6 \\
Occipital lobe & 1.1 & 0.3 & & 0.9 & 1.6 \\
Parietal lobe & 0.1 & 0.0 & & 0.0 & 0.1 \\
Cerebellum & 0.7 & 0.1 & & 0.4 & 0.4 \\
\hline
\end{tabular}

(b) Contusions in patients with predominantly frontal or occipital fractures

\begin{tabular}{lll}
\hline Site of fracture & $M C I$ & \\
\cline { 2 - 3 } & Frontal & Occipital \\
\hline Frontal $(\mathrm{n}=23)$ & $7 \cdot 9$ & $2 \cdot 7$ \\
Occipital $(\mathrm{n}=44)$ & $7 \cdot 5$ & $2 \cdot 1$ \\
\hline
\end{tabular}

Table 3 MTCI in patients with and without fracture of the skull, lucid interval, and diffuse damage to white matter (from Adams et al. ${ }^{12}$ )

\begin{tabular}{|c|c|}
\hline $\begin{array}{l}\text { With a fracture }(n=120) \\
19 \cdot 1 \\
\text { With a lucid interval }(n=58) \\
15 \cdot 1 \\
\text { With diffuse damage to } \\
\text { white matter }(n=19) \\
\quad 7 \cdot 8\end{array}$ & $\begin{array}{l}\text { Without a fracture }(n=31) \\
66 \cdot 7 \\
\text { Without a lucid interval }(n=93) \\
17 \cdot 5 \\
\text { Without diffuse damage to } \\
\text { white matter }(n=132) \\
\quad 17 \cdot 8\end{array}$ \\
\hline
\end{tabular}

patients with localising fractures as defined above and in the 67 patients with predominantly frontal or occipital fractures in Table 2 , and a comparison between the MTCIs in patients with and without skull fracture, lucid interval, ${ }^{13}$ and diffuse damage to white matter as defined below is given in Table 3.

Statistical assessment of the results, using a nonparametric test, discloses that in the present series contusions were more severe in patients with a fracture of the skull $(P<0.001)$ and less severe in patients with diffuse damage to white matter $(P<0.001)$. There is no evidence to suggest that there is any difference in the severity of the contusions in patients who do or do not experience a lucid interval after head injury. In the 30 patients with lateralising fractures, there is no evidence that contusions are more severe in the cerebral hemisphere contralateral to the fracture, while in patients with predominantly frontal or occipital fractures contusions are more severe in the frontal lobes than in the occipital lobes.

Of the 15 cases with severe contusions defined above, the age range was 9 to 65 years and the duration of survival ranged from less than 12 hours to seven days. Of these 15 cases, 10 were unconscious from the time of injury until death, and five were lucid ( 3 partial and 2 total). A fracture of the skull was present in every case, and in 13 there was evidence of raised intracranial pressure. In only three of these cases were there no other types of severe brain damage, and each case had a particularly extensive depressed fracture of the skull.

\section{Diffuse damage to white matter}

The cases in the present series with this type of brain damage have been described in detail elsewhere ${ }^{10}$ where the relevant literature is also reviewed. The hallmarks of this type of brain damage that can be seen macroscopically are focal lesions in the corpus callosum and in the dorsolateral quadrants of the rostral brain stem. In patients who die soon after their injury these lesions are usually haemorrhagic (Figs 6 and 7), but if the patient survives for some weeks or longer these lesions come to be represented by shrunken, sometimes cystic, and usually small 


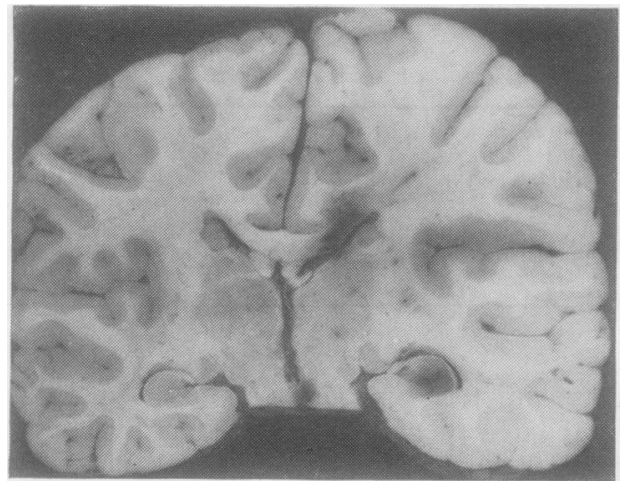

Fig. 6 Diffuse damage to white matter (six-day survival after injury): there is a recent haemorrhagic lesion in the corpus callosum. Compare with Fig. 8 (from Adams ${ }^{8}$ ).

scars (Figs 8 and 9). In patients who survive for many months after their injury, there is characteristically some loss of bulk of the white matter of the cerebral hemispheres and an associated increase in the size of the ventricles (Fig. 10). Histological examination will disclose widespread evidence of damage to nerve fibres, as shown by the presence of axonal retraction balls, particularly in the corticospinal tracts and medial lemnisci, in short survivors (Fig. 11), multiple microglial stars (Fig. 12) in those of intermediate survival, and long tract degeneration stainable by the Marchi technique in patients who survive for more than two or three months (Fig. 13). Other common abnormalities associated with this type of brain damage are small petechial haemorrhages throughout the brain, which are presumably attributable to the rupture of small blood vessels, and linearly

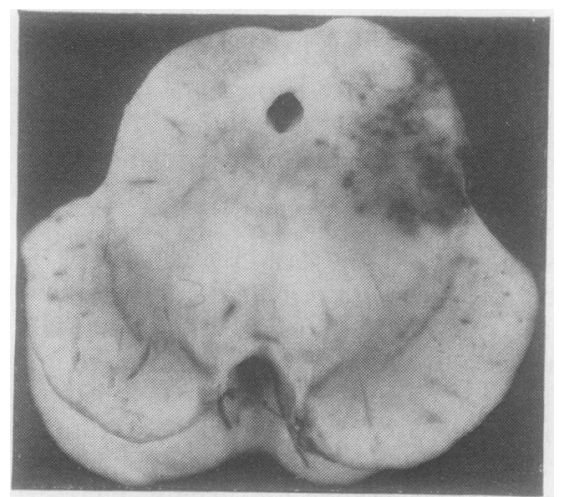

Fig. 7 Diffuse damage to white matter (12-day survival after injury): there is a recent haemorrhagic lesion in the dorsolateral quadrant of the rostral brain stem. Compare with Fig. 9 (from Adams ${ }^{8}$ ).

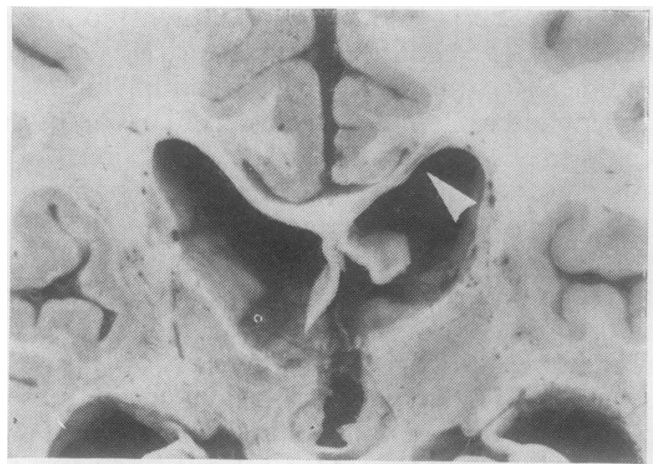

Fig. 8 Diffuse damage to white matter (21-month survival after injury): there is a shrunken cystic area (arrow) in the corpus callosum. The corpus callosum is also thin (from Adams et al. ${ }^{10}$ ).

disposed haemorrhagic lesions in the subcortical white matter at the dorsal angles of the cerebral hemispheres, particularly in the posterior frontal and parietal regions.

In the present series there were 19 patients with diffuse damage to white matter: all were unconscious from the moment of impact, that is, none experienced a lucid interval, and remained so or in a vegetative state $^{18}$ until death. The duration of survival ranged from 12 hours to 21 months. In eight of these cases there was no neuropathological evidence of there having been a significantly high intracranial pressure during life; in this group there was a fracture of the skull in only one $(12.5 \%)$ patient, and the

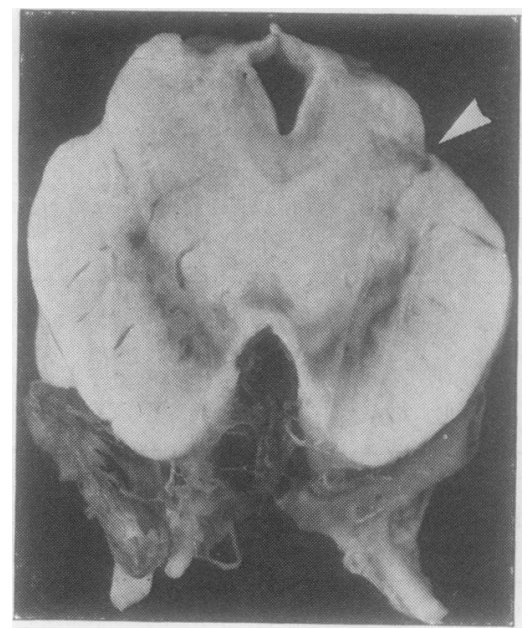

Fig. 9 Diffuse damage to white matter (nine-month survival after injury): there is a shrunken cystic area (arrow) in the dorsolateral quadrant of the rostral brain stem (from Adams et al. ${ }^{10}$ ). 


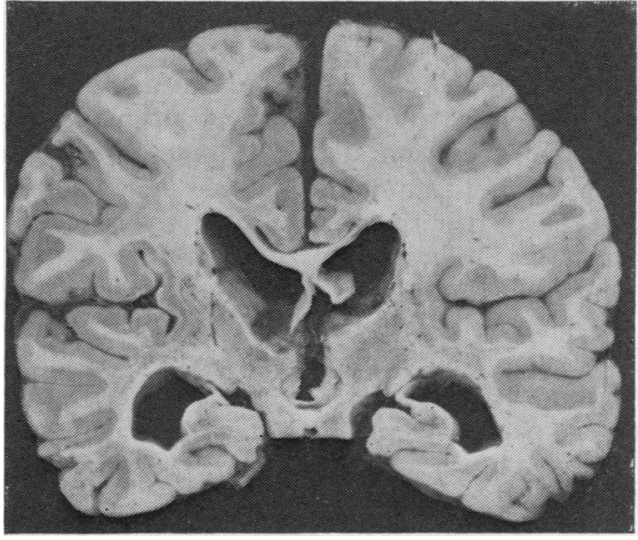

Fig. 10 Diffuse damage to white matter (21-month survival after head injury): there is a reduction in the bulk of the white matter and enlargement of the ventricular system (from Adams et al. ${ }^{10}$ ).

MTCI was low-3.9. In the remaining 11 the intracranial pressure had been high during life because of a complicating expanding lesion; there was a fracture of the skull in five $(45 \%)$ of the group and the MTCI was $10 \cdot 7$.

Thus in this group of 19 cases there was a much lower incidence of lucid interval than in the rest of the series $(0 \%$ compared with $44 \%$; $><0.001)$, a high intracranial pressure $(58 \%$ compared with $87 \% ; P<0.005)$, and fracture of the skull $(32 \%$ compared with $86 \% ; \mathrm{P}<0.001)$. Contusions were also considerably less severe (MTCI of $7 \cdot 8$ compared with 17.8 for the rest of the series; $P<0.001$ ).

Other types of diffuse brain injury

In the series of 151 cases there were two young adults, each of whom was unconscious from the

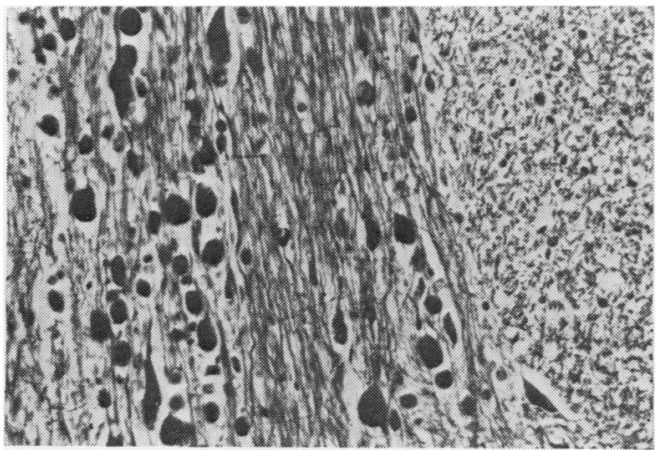

Fig. 11 Diffuse damage to white matter (6-day survival after injury): there are numerous axonal retraction balls in the basis pontis (from Adams et al. ${ }^{10}$; Palmgren $\times 200$ ).

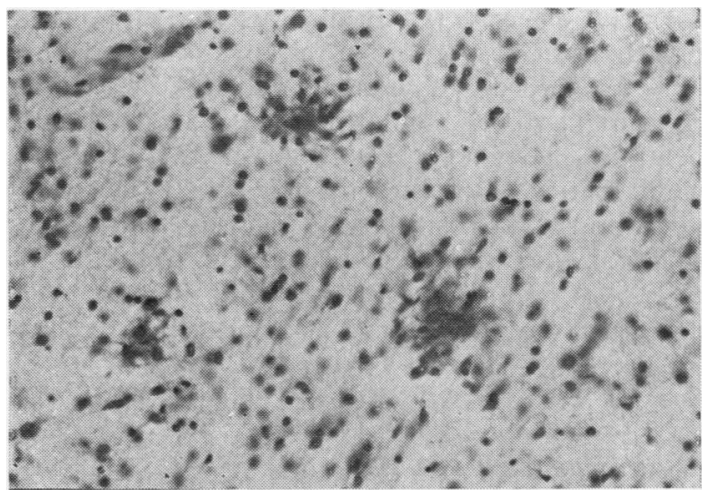

Fig. 12 Diffuse damage to white matter (six-week survival after injury): there are numerous clusters of microglia in the white matter (from Adams et al. ${ }^{10}$; cresyl violet $\times 250$ ).

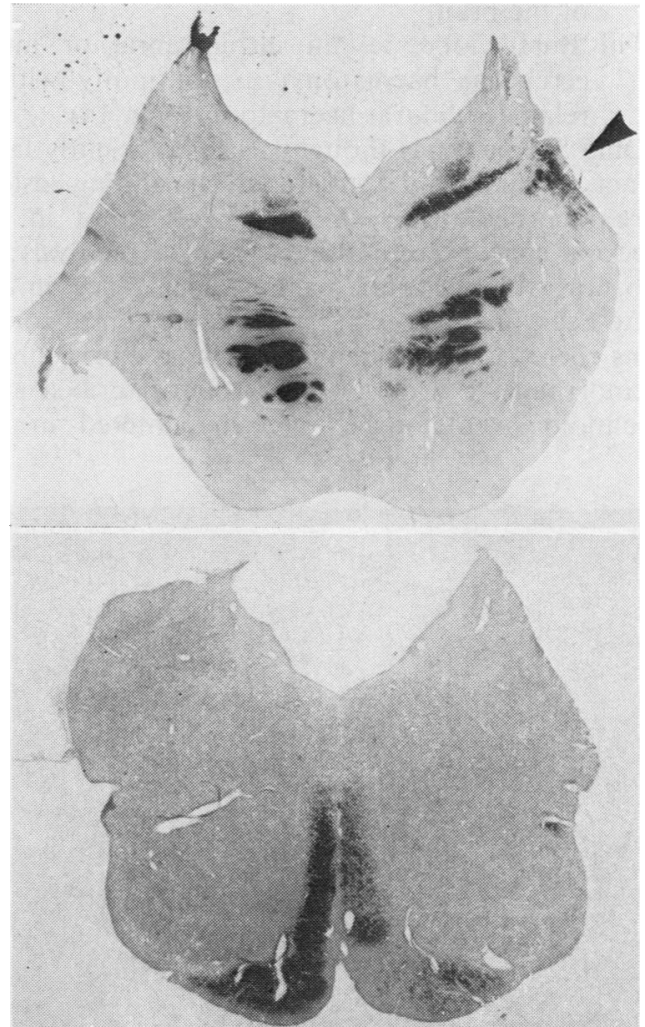

Fig. 13 Diffuse damage to white matter (21-month survival after injury): there is long tract degeneration in the corticospinal tracts and in the medial lemnisci. There is also a focal abnormality (arrow) in the dorsolateral quadrant of the brain stem (compare with Figs 7 and 9). Marchi preparation. 
moment of impact and died within 12 hours of injury. Both had a fracture of the skull, no histological evidence to suggest that the intracranial pressure had been high during life, and innumerable petechial haemorrhages throughout the cortex and white matter of the cerebral hemispheres and in the mid-brain of the rostral pons. The petechial haemorrhages in the cortex were construed as superficial but extensive contusions, with the result that the MTCI in these two cases was high -41 and 55 , respectively.

\section{PRIMARY COMPLICATIONS}

\section{Intracranial haemorrhage}

This was classified as follows:

(a) Extradural haematoma

(b) Intradural haematoma

i Subdural haematoma

ii Discrete intracerebral or intracerebellar haematomas not in continuity with the surface of the brain

iii 'Burst' lobe, ie, an intracerebral or intracerebellar haematoma in continuity with a related subdural haematoma (Fig. 14).

Only haematomas thought to be sufficiently large to act as significant intracranial expanding lesions were recorded. Thus thin films of blood in the subdural space were excluded from the analysis; so also was subarachnoid haemorrhage since in any acute head injury in which there are cerebral contusions there is almost inevitably some subarachnoid haemorrhage. Furthermore, no circumscribed subarachnoid haematomas were encountered in the

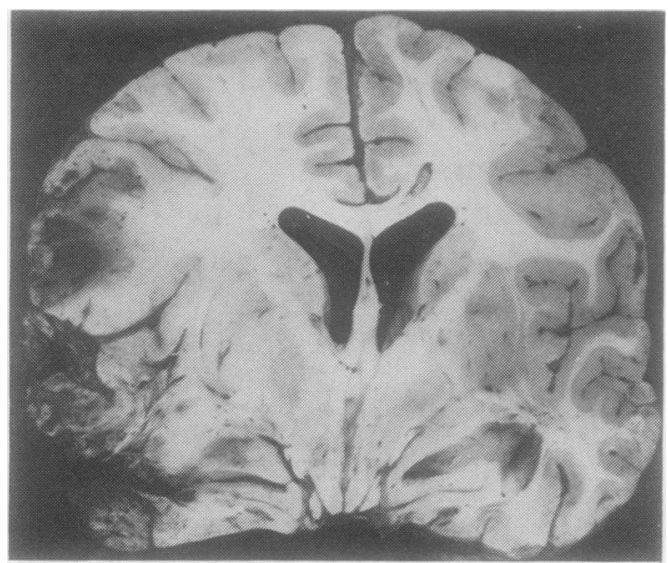

Fig. 14 Intracranial (intradural) haematoma (three-day survival after injury): the haematoma in the left temporal lobe was in continuity with an acute subdural haematoma ('burst' lobe). The lesion adjacent to the corpus callosum to the right of the mid-line is a ventricular catheter track. present series although they do occasionally occur. The great majority of the haematomas recorded had been evacuated surgically before death.

Ninety-seven patients had intracranial haematomas; they were supratentorial in 88 , infratentorial in five, and both supratentorial and infratentorial in four. The distribution of the supratentorial haematomas was as follows:

Extradural haematoma was present in 16 patients. In six there were no other intracranial haematomas, and in one of these six patients there were bilateral extradural haematomas. In the 10 other patients with extradural haematoma there were also intradural haematomas-seven with ipsilateral supratentorial subdural haematomas ( 2 cases) or intracerebral haematomas (5 cases), one with a contralateral 'burst' temporal lobe, and two with haematomas in the posterior fossa (one patient with a subdural haematoma and one with both extradural and intracerebellar haematomas). There was a fracture of the skull in relation to the extradural haematoma in 15 of the 16 patients. Of the 16 patients with extradural haematomas, eight experienced a lucid interval-three of the six patients with an extradural haematoma only and four of the 10 patients with an intradural haematoma as well.

Subdural haematoma occurred in 30 patients. Ten of these patients experienced total lucid intervals and seven partial lucid intervals. Six patients with subdural haematoma did not have a fracture of the skull. Of the 51 patients with lateralising fractures, 10 had subdural haematomas. In five of these the haematoma was contralateral to the fracture.

One of the 30 patients with subdural haematoma had an ipsilateral extradural haematoma, two had contralateral 'burst' lobes (one frontal and one temporal), while two had contralateral frontal intracerebral haematomas.

Intracerebral haematoma was found in 33 patients. There were 46 discrete haematomas, 23 in the frontal lobes, 13 in the temporal lobes, four in the parietal lobes, four centred on the basal ganglia, and two in the occipital lobes. Twenty-three patients had single haematomas, eight had two, one had three, and one had four. Five of the 33 patients also had an extradural haematoma and two a subdural haematoma. Seven patients had a total lucid interval, eight a partial lucid interval, and 18 no lucid interval. Four of the 33 patients did not have a fracture of the skull.

'Burst lobes' were present in 33 patients, 15 being frontal and 19 temporal. One patient had 'burst' frontal and temporal lobes. A contralateral subdural haematoma was present in two patients and an ipsilateral extradural haematoma in one. There was no fracture of the skull in three of the 33 patients. 


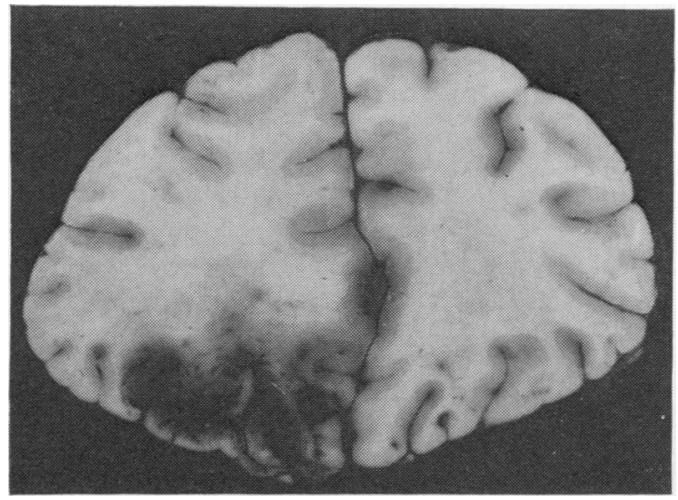

Fig. 15 Brain swelling (24-hour survival after injury): there is swelling of the white matter adjacent to contusions affecting the orbital surface of the left frontal lobe.

Of the total of 33 , four experienced a total lucid interval, nine a partial, and 20 no lucid interval.

In the 51 patients with lateralising fractures there were 17 with 'burst lobes'. Of these, nine were ipsilateral to the fracture of the skull and eight contralateral.

Infratentorial haematomas occurred in nine casesthree intracerebellar haematomas, two subdural haematomas, two 'burst lobes', and two extradural haematomas. Four of these cases had supratentorial haematomas as well. There was a fracture of the

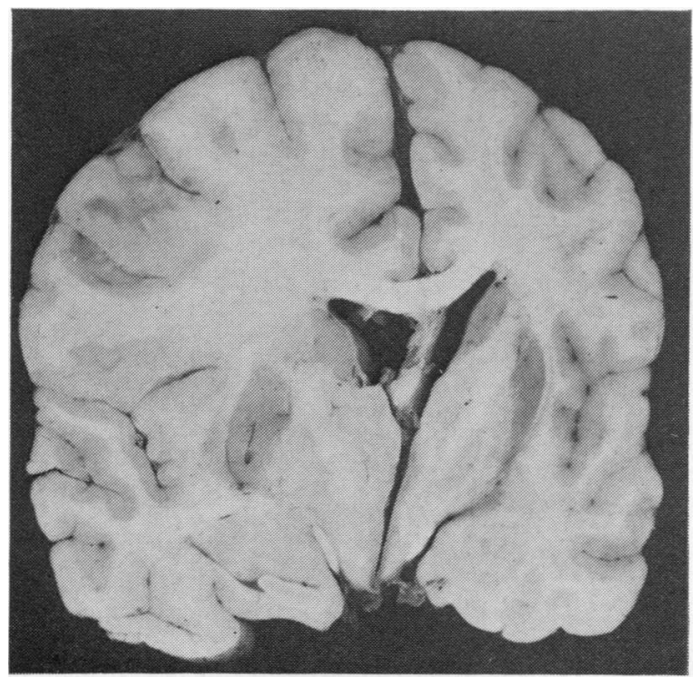

Fig. 16 Brain swelling (10-day survival after injury): there is diffuse swelling of the left cerebral hemisphere and a shift of the mid-line structures to the right.

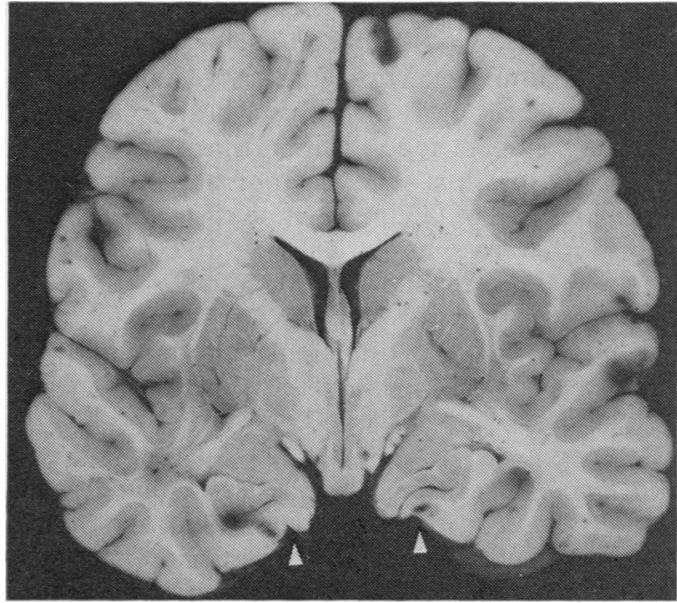

Fig. 17 Brain swelling (24-hour survival after injury): there is diffuse swelling of both cerebral hemispheres. The lateral ventricles are small and symmetrical, and there is bilateral tentorial herniation (arrows).

skull implicating the posterior fossa in six of these nine cases, and it was unilateral to the haematoma in every case.

\section{Brain swelling}

Brain swelling is used in preference to 'cerebral oedema' since cerebral vasodilatation may produce swelling without there being any increase in water content.

Localised brain swelling occurs adjacent to cerebral contusion (Fig. 15) and may therefore contribute to any increase in intracranial pressure.

Unilateral brain swelling, that is, diffuse swelling of one cerebral hemisphere (Fig. 16), was present in 17 patients. There was a fracture of the skull in every case. Of the 51 cases with lateralising fractures there was unilateral brain swelling in five. Swelling was ipsilateral to the fracture in two and contralateral in three. The contusion index for the ipsilateral hemisphere was $11 \cdot 8$, and for the contralateral hemisphere $4 \cdot 3$.

In seven of the 17 cases with unilateral brain swelling an ipsilateral acute subdural haematoma had been evacuated; this association just attains statistical significance $(P<0.025)$ in comparison with cases with unilateral brain swelling without a subdural haematoma.

Bilateral brain swelling, that is, diffuse swelling of both cerebral hemispheres (Fig. 17), was seen in nine patients. Six of these cases were aged 16 or under, and there was no fracture of the skull in four. One patient, a child aged 4 , who developed an extradural 
haematoma which was evacuated, had a complete lucid interval. Two others had partial lucid intervals. Of the remaining six who did not have lucid intervals, two were examples of diffuse brain damage of immediate impact type, while a third came into the category of multiple small haemorrhages throughout the cortex. The MTCI in the nine cases was $20 \cdot 9$, and contusions were symmetrical (left 10.0; right 10.9). For the five patients aged 15 years or less the MTCI was 11.2; in three of these five it was 0,2 , and 3 respectively, that is, contusions were minimal.

\section{SECONDARY COMPLICATIONS}

Brain damage secondary to raised intracranial pressure It was difficult in the past for the neuropathologist to decide whether or not a patient had experienced a high intracranial pressure during life since, during the period of spatial compensation when the intracranial pressure is not significantly high, there can be considerable brain shift and the development of internal herniae. ${ }^{19}$ As a result of the introduction of continuous monitoring of intracranial pressure, however, we ${ }^{17}$ have been able to define certain criteria which allow the neuropathologist to state with a considerable degree of certainty if a supratentorial expanding lesion has been sufficiently large to elevate the intracranial pressure during life even when it has not been monitored clinically. The principal criterion is pressure necrosis in one or both parahippocampal gyri (Figs 18-20). This necrosis

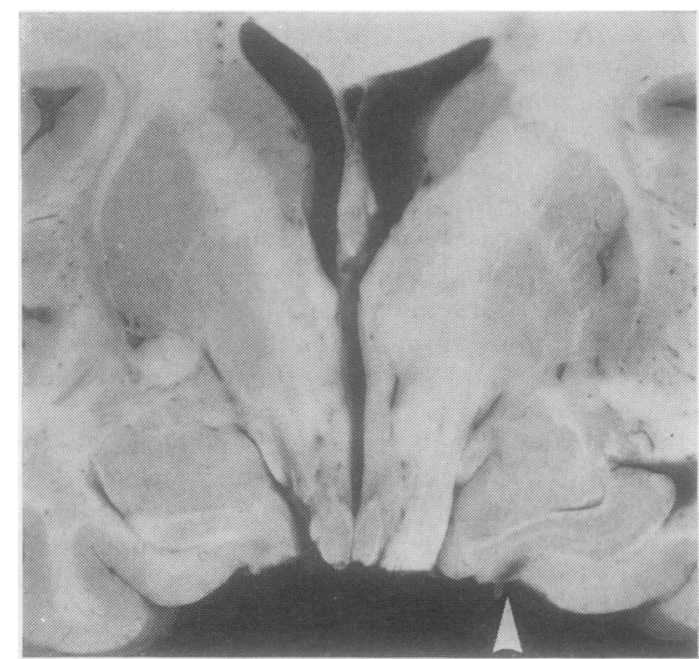

Fig. 18 Raised intracranial pressure: there is a small wedge of haemorrhagic pressure necrosis (arrow) in the right parahippocampal gyrus along the line of a tentorial hernia.

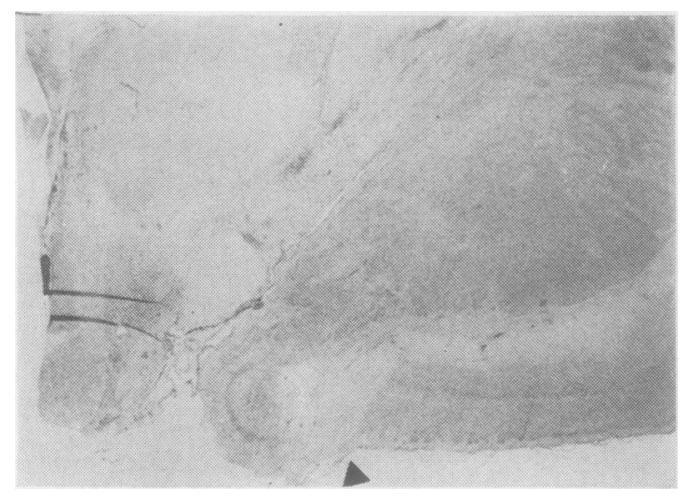

Fig. 19 Raised intracranial pressure: there is a wedge of pale pressure necrosis (arrow), which could not be seen macroscopically, in the right parahippocampal gyrus (from Adams and Graham $^{17}$ ).

can probably be equated with a pressure differential between the supratentorial and infratentorial compartments. The criteria defined, however, have two possible fallacies: if the intracranial pressure is high because of an infratentorial expanding lesion there will be no tentorial herniation and therefore no pressure necrosis in the parahippocampal gyrus; and if the pressure has been high for only a very short period of time, the latter may be insufficient to allow the occurrence of even localised tissue necrosis. Necrosis in the parahippocampal gyrus is therefore indicative of a high intracranial pressure, probably

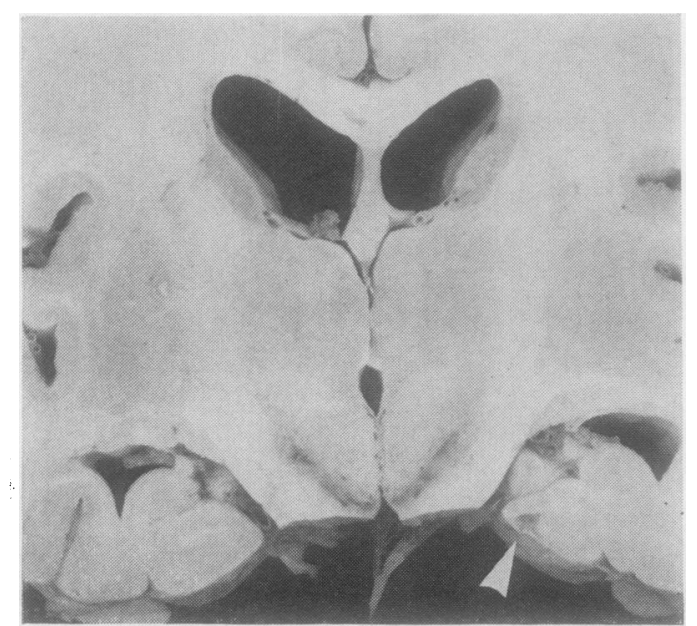

Fig. 20 Raised intracranial pressure: there is a small old cystic lesion (arrow) in the right parahippocampal gyrus indicative of previous raised intracranial pressure and tentorial herniation (from Adams and Graham ${ }^{17}$ ). 
of at least some hours' duration, caused by a supratentorial expanding lesion.

Of the 151 cases there was evidence of a high intracranial pressure in $125(83 \%)$ patients. The causes were usually intracranial haematoma or brain swelling. Thus brain damage-usually haemorrhage or infarction in the brain stem-secondary to a high intracranial pressure and shift and herniation of the brain was an important factor leading to death. This type of damage was, however, not a common cause of the vegetative state since only five of the 125 patients survived for more than four weeks after their injury and, of these, two had diffuse damage to white matter and two severe hypoxic brain damage in addition. In none of the five cases was there severe brain stem damage secondary to the high intracranial pressure.

Of the 26 cases with no evidence of the intracranial pressure having been high during life, eight had evidence of diffuse damage to white matter, seven had severe hypoxic brain damage, and two had multiple small haemorrhages throughout the brain. The causes of death in the remainder were cardiac and/or renal failure (3), meningitis (2), fulminating fat embolism (1), a high cervical spinal injury (1), and severe chest injury (1). One patient had an isolated extradural haematoma in the posterior fossa.

\section{Hypoxic brain damage}

The incidence and distribution of this type of brain damage in the present series have been described in detail elsewhere ${ }^{11}$ where the relevant literature is also reviewed. Of the total of 151 cases, there was hypoxic damage in the Ammon's horns in $122(80 \%)$, in the basal ganglia in $119(79 \%)$, in the cerebral cortex-excluding infarction in the medial occipital cortex secondary to a high intracranial pressure and tentorial hernia -in $70(46 \%)$, and in the cerebellum in $67(44 \%)$. There were only 13 cases in the entire series in which there was no hypoxic brain damage.

The hypoxic damage in the cerebral cortex took several forms; in the commonest type (33 cases) it was centred on the boundary zones (Fig. 21) between the major cerebral arterial territories, particularly between the anterior and middle cerebral arterial territories, but also in the lateral part of the occipital lobe in the common boundary zone of the anterior, middle, and posterior cerebral arterial territories. The hypoxic damage was bilateral in 27 of these 33 cases. In another group of 14 cases the hypoxic damage was centred on the territories supplied by the anterior and/or the middle cerebral arteries. This type of damage was bilateral in eight. In 10 cases there were multiple foci of necrosis throughout the cerebral cortex, while in eight there was severe and diffuse necrosis of the cortex (Fig. 22)

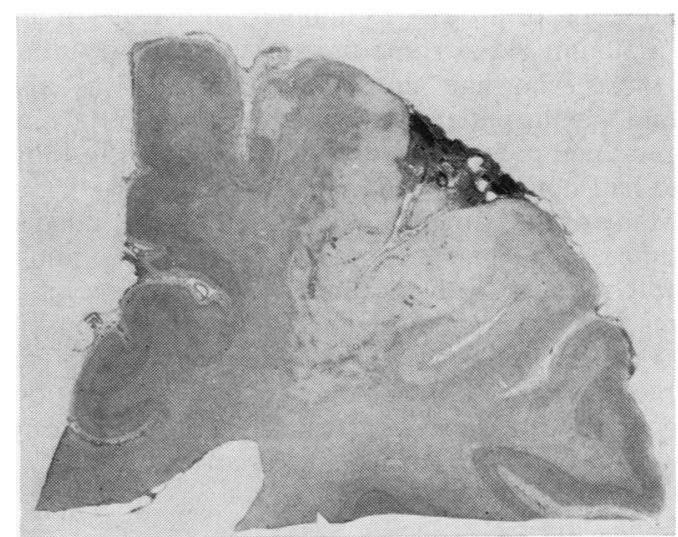

Fig. 21 Hypoxic brain damage (eight-day survival after injury): there is a large, mainly pale, wedge-shaped infarct in the boundary zone between the territories supplied by the right anterior and middle cerebral arteries (from Graham et al. ${ }^{11}$; celloidin section, cresyl violet).

attributable to an episode of cardiac arrest or status epilepticus. In five the pattern of hypoxic damage was mixed in type. The cortical damage was assessed as being severe in 29 cases $(41 \%$ of the cases with hypoxic damage in the cortex), moderately severe in $35(50 \%)$, and mild in six $(9 \%)$.

Hypoxic brain damage was more common in patients who had sustained a known clinical episode of hypoxia (a systolic blood pressure of less than

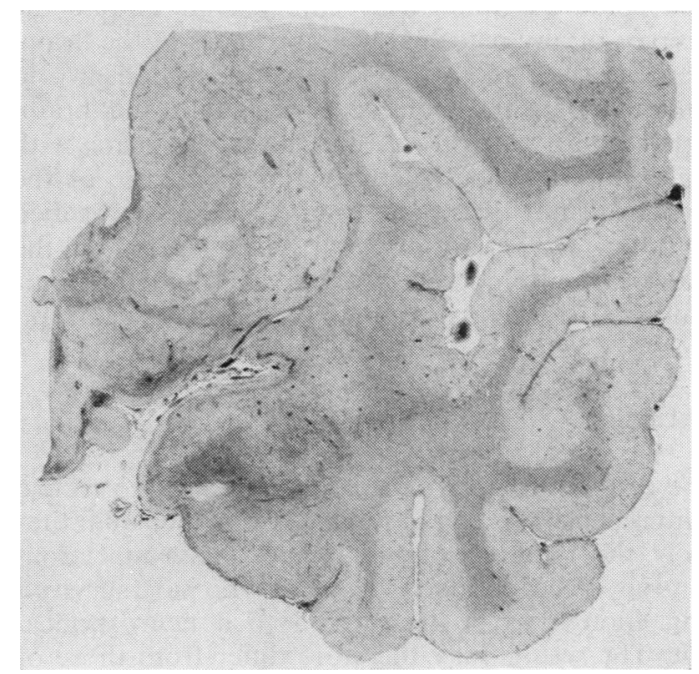

Fig. 22 Hypoxic brain damage (four-day survival after injury): there is pallor of the cortex indicative of massive neuronal necrosis. This was not apparent macroscopically (from Graham et al. ${ }^{11}$; celloidin section, cresyl violet). 
$80 \mathrm{~mm} \mathrm{Hg}$ for at least 15 minutes, or a $\mathrm{PaO}_{2}$ of less than $50 \mathrm{~mm} \mathrm{Hg}$ at some time after the injury) than in those who had not $(P<0.005)$. It was also commoner in patients who had experienced a high intracranial pressure as defined above than in those who had not $(\mathrm{P}<0.005)$.

Many of the patients in this study were included in another study in which the angiographic findings were correlated with the presence of hypoxic brain damage. ${ }^{20}$ This established a significant relationship between arterial spasm alone, the presence of an intracranial haematoma alone, or their combination, and hypoxic damage-particularly of arterial territory distribution in the ipsilateral cortex.

\section{Infection}

Meningitis occurred in four of the 151 cases and in each of these there was a fracture of the base of the skull. Meningitis developed within seven days of injury in three of the cases but in the fourth it did not occur until four weeks after the injury.

\section{OTHER ABNORMALITIES IDENTIFIED IN THE BRAIN}

There were two cases in the series with fulminating fat embolism. One of these was a girl aged 19 with a small fracture of the base of the skull and minimal cerebral contusions who was lucid a few minutes after her original injury. She also had a fracture of the femur. Her conscious level began to deteriorate a few hours after injury but no expanding lesion was identifiable radiologically to account for this. She died some 30 hours after injury and, despite the absence of any petechial haemorrhages in the brain, there was severe and extensive fat embolism and innumerable small infarcts throughout the brain. The second patient-a man aged 24 years with multiple long bone fractures and a fracture of the skull-did not experience a lucid interval. He died 44 hours after his injury, and examination of the brain showed moderately severe cerebral contusions and extensive hypoxic damage; there were again no petechial haemorrhages in the white matter.

\section{Discussion}

One of the principal aims of this paper is to record comprehensively and, as far as possible, quantitatively the types of brain damage that result from non-missile head injury in patients who have survived long enough to be transferred to a neurosurgical unit. The results may therefore differ from those of series based on the examination of the brains of individuals who die very soon after head injury or who die in district general hospitals. They should, however, serve to emphasise the types of brain damage that occur at impact, and over which the clinician inevitably has little or no control, and the complications leading to secondary brain damage over which he may have - at least potentially - some control. A particularly important factor in this respect is delay in recognising the development of intracranial haematoma. ${ }^{21} 22$ Furthermore, the types of brain damage that we have encountered are likely to be the pathological substrate in patients who remain permanently brain-damaged after head injury.

The second aim is to impress on pathologists that severe brain damage in patients dying as a result of a head injury may well be missed at necropsy unless various lesions are looked for specifically. This task is, of course, made much easier if the brain is fixed before dissection-indeed unilateral or diffuse brain swelling is particularly difficult to identify if the brain is sliced in the unfixed state. It is, however, usually possible to diagnose diffuse damage to white matter macroscopically provided the rostral brain stem and the corpus callosum are examined at several levels, but recent hypoxic brain damageeven when severe and extensive-may be identifiable only histologically. ${ }^{14-16}$ It is therefore easy for a pathologist to attribute death to a fracture of the skull and to contusions although neither may have contributed materially to the fatal outcome.

The adoption of a quantitative contusion index has for the first time allowed of a statistical approach to the assessment of contusions. Thus we have been able to confirm that their distribution and severity accords with subjective impression. We have also demonstrated that contusions are significantly more severe in patients with a fracture of the skull than in those without a fracture. It is also clear that the concept of contre coup, that is, that brain damage is selectively severe diametrically opposite to the site of injury, cannot be interpreted too rigidly; thus in the cases with lateralising fractures associated with a corresponding scalp lesion contusions were not more severe in the contralateral hemisphere, and in patients with severe frontal fractures, frontal contusions were more severe than contusions in the occipital lobes. Another observation suggesting that contusions per se are of limited importance is that in 20 of the 151 cases in the series they were trivial (a CI of not more than 1 in any of the anatomical locators assessed). There were, however, three patients in whom severe contusions seemed to be the principal factor leading to a fatal outcome, since in each case they were the only evidence of severe brain damage; in every one of these cases there was an extensive depressed fracture of the skull. The fact that there is no correlation between the severity of contusions and a lucid interval is probably not surprising since 
contusions are indicative of focal damage to the brain.

Diffuse damage to white matter is probably the most important single factor governing the outcome in a patient with head injury since it occurs at the moment of impact and is therefore neither preventable nor reversible in the course of further clinical management. None of the 19 patients with this type of brain damage experienced a lucid interval, and there were significantly fewer cases with a fracture of the skull, contusions, and a high intracranial pressure compared with the remainder of the series. Indeed, the brains of patients with this type of brain damage may appear virtually normal externally. This is the commonest type of brain damage we observe in brains submitted to us by pathologists in other hospitals because they have found it difficult to reconcile the clinical picture of severe brain damage resulting from a head injury with the external appearance of the brain. It is also, in our experience, the commonest cause of the vegetative state after head injury. As we have discussed before, ${ }^{10}$ there has been some controversy in the past as to the pathogenesis of this type of brain damage but since it clearly occurs at the moment of impact it seems likely that it is brought about by shear-strains engendered in the white matter which are sufficiently intense to cause disruption of nerve fibres. Rotational movements are probably of greater importance than translational forces in its pathogenesis. Although the clinical picture may be that of primary brain stem damage, damage is never restricted to the brain stem. The C-T scan is often normal ${ }^{23}$ in patients with diffuse damage to white matter but it is sometimes possible to identify the focal lesions in the corpus callosum and in the brain stem. ${ }^{24}$

The two cases with multiple small haemorrhages throughout the cortex are difficult to classify. Since both patients were unconscious until they died within 12 hours of injury, it seems likely that this is another type of diffuse immediate impact injury. It may be commoner than the present series suggests since we encounter very few patients who die within 12 hours of injury.

Intracranial haematoma is a common and wellrecognised complication of head injury. ${ }^{22} 25$ It is perhaps worth emphasising that only $50 \%$ of the patients with extradural haematoma experienced a lucid interval, that $57 \%$ of the patients with a pure subdural haematoma did experience a lucid interval, and that in $50 \%$ of the patients with a lateralising fraciure and a subdural haematoma the haematoma was contralateral to the fracture.

Brain swelling is an important complication of head injury since it may lead to a considerable increase in the volume of the intracranial contents. It is of particular significance in two contexts- diffuse swelling of one cerebral hemisphere and diffuse swelling of both cerebral hemispheres. The occurrence-or perhaps persistence-of swelling of a cerebral hemisphere after evacuation of an ipsilateral acute subdural haematoma is generally recognised but the association just attained statistical significance in the present series. Unilateral oedema is not restricted to patients with subdural haematoma, and in such cases contusions were more severe in the ipsilateral hemisphere. The occurrence of diffuse brain swelling in children is also well recognised, ${ }^{2326}$ and six of the nine patients in the present series with diffuse brain swelling were aged 16 years or less. That this can occur in the absence of severe contusions is clearly established by the fact that in three of the children in the present series with diffuse brain swelling the MTCI was very low. The pathogenesis of brain swelling in association with acute head injury has not been clearly defined but it seems possible, at least in children, that it may be contributed to by loss of vasomotor tone, which in turn leads to vasodilatation. ${ }^{26}{ }^{27}$ Vasodilatation itself will produce brain swelling, and if the vasodilatation persists the blood-brain barrier may become defective, this in turn leading to true vasogenic extracellular cerebral oedema. Other causes of diffuse brain swelling are diffuse damage to white matter and severe hypoxic brain damage.

Of the secondary complications of head injury, brain damage secondary to a high intracranial pressure and distortion and herniation of the brain is well recognised, and analysis of the present series does not yield any new information. It is, however, worth emphasising that, despite the frequency of secondary damage to the brain stem, this was not a common cause of prolonged coma since every patient with severe secondary brain stem damage died within four weeks of injury.

It is also clear from the present analysis that hypoxic brain damage is common in patients dying as a result of a head injury. Perhaps the principal reason why this high incidence has not been emphasised in the past is that much of it can be identified only microscopically. ${ }^{14-16}$ Furthermore, the incidence may be particularly high in the present series since all the patients survived long enough to be transferred to a neurosurgical unit. We have already argued $^{11}$ that it is unlikely that the hypoxic damage occurs at the moment of impact and that the damage may therefore be potentially preventable since it is significantly more common in patients known to have experienced an episode of hypoxia in the postinjury period and in patients in whom the intracranial pressure has been high. From the distribution of the brain damage it seems likely that much of it is due to a reduction in cerebral perfusion pressure-hence 
the need to preserve cerebral blood flow and arterial oxygenation in patients who have sustained a head injury. Severe hypoxic brain damage in a patient with head injury can also be associated with a normal C-T scan. ${ }^{23}$

The distribution of hypoxic damage is most easily assessed in large histological sections. It is hardly feasible for a general pathologist to undertake a comprehensive neurohistological analysis in every case of fatal head injury but it is usually possible to establish that a patient has experienced an episode of hypoxia sufficiently severe to produce brain damage by the histological examination of quite small, bilateral blocks from the 'selectively vulnerable areas', viz, the arterial boundary zones, the Ammon's horns, the thalamus, and the cerebellum. ${ }^{15} 16$

Meningitis is another well recognised complication of a head injury, and it occurred in four cases in the present series. In every case there was a fracture of the base of the skull, and in one of the cases meningitis did not develop until four weeks after the injury.

There were two cases in the present series with fulminating fat embolism. This was the only other type of severe brain damage encountered, and in each case it was entirely unexpected since there was no macroscopic evidence suggestive of cerebral fat embolism.

This series of cases forms part of a systematic study of the pathology of brain damage that occurs in fatal non-missile head injury and is associated with the MRC Head Injury Research Programme being undertaken in the University Department of Neurosurgery. That study has been recording for 11 years clinical data about all severe head injuries in this Institute as well as cases from centres in other parts of the world. ${ }^{28}$ The cases reported in the present series were collected some 10 years ago, so that a study of more recent cases might reveal a different incidence of brain damage since changing patterns of admission and management may have produced some alterations in the types and distribution of secondary complications. A computerised data collection system has now been developed so that as close clinicopathological correlations as possible can be established. Special attention is being paid to factors that might have been avoided, ${ }^{29} 30$ particularly in patients who were known to have talked some time after their injury. ${ }^{13}$ These studies have highlighted the importance of hypoxia and hypotension, and of reducing delay in the treatment of intracranial haematoma. These factors have all been implicated as events leading to a fatal outcome, and it seems likely that they are also factors contributing to persistent brain damage in patients who survive their injury.
The present study would not have been possible without the willing co-operation of the forensic pathologists in the West of Scotland who allowed us to fix the brains intact before dissection. We are particularly indebted to Emeritus Professor G Forbes, Professor WA Harland, Dr A Dick, Dr WDS McLay, Dr AA Watson, and Dr WP Weir. We are also indebted to the staff of the Department of Neurosurgery for access to their clinical records. It is a pleasure to thank the Department of Medical Illustration of the Southern General Hospital for their help in preparing the illustrations, and Mrs J Rubython for typing the manuscript.

We are grateful to the editors of Brain and to the Oxford University Press for permission to reproduce Figs $1,2,8,9,10,11$, and 12 ; to the editor of Neuropathology and Applied Neurobiology and Blackwells Scientific Publications for permission to reproduce Figs 3, 4, 5, 19, and 20 and Tables 1, 2, and 3 ; to the North-Holland Publishing Company for permission to reproduce Figs 6 and 7; and to the editor of the Journal of the Neurological Sciences and Elsevier/North-Holland Biomedical Press for permission to reproduce Figs 21 and 22 .

LSP was supported by MRC grant G976/491/N.

\section{References}

${ }^{1}$ Lindenberg R, Freytag E. The mechanism of cerebral contusions. Arch Pathol (Chic) 1960;69:440-69.

2 Freytag E. Autopsy findings in head injuries from blunt forces. Arch Pathol 1963;75:402-13.

${ }^{3}$ Maloney AFJ, Whatmore WJ. Clinical and pathological observations in fatal head injuries: a 5-year survey of 173 cases. Br J Surg 1969;56:23-31.

4 Strich SJ. The pathology of brain damage due to blunt head injuries. In: Walker AE, Caveness WF, Critchley $\mathrm{M}$, eds. The Late Effects of Head Injury. Springfield, Ill.: Charles C Thomas, 1969;501-24.

${ }^{5}$ Lindenberg $R$. Trauma of meninges and brain. In: Minckler J, ed. Pathology of the Nervous System. Vol. 2. New York: McGraw-Hill, 1971;1705-65.

- Sevitt S. Fatal road accidents in Birmingham: times to death and their causes. Brit J Acc Surg 1973;4:281-93.

${ }^{7}$ Hardman JM. The pathology of traumatic brain injuries. In: Thompson RA, Green JR, eds. Advances in Neurology. Vol. 22. New York: Raven Press, 1979;15-50.

${ }^{8}$ Adams JH. The neuropathology of head injuries. In: Vinken PJ, Bruyn GW. Handbook of Clinical Neurology. Vol. 23. Amsterdam: North-Holland Publishing Company, 1975;35-65.

- Mitchell DE, Adams JH. Primary focal impact damage to the brain-stem in blunt head injuries: does it exist? Lancet 1973 ;ii:215-8.

${ }^{10}$ Adams JH, Mitchell DE, Graham DI, Doyle D. Diffuse brain damage of immediate impact type. Its relationship to 'primary brain-stem damage' in head injury. Brain 1977;100:489-502.

${ }^{11}$ Graham DI, Adams JH, Doyle D. Ischaemic brain damage in fatal non-missile head injuries. $J$ Neurol Sci 1978; 39:213-34. 
12 Adams JH, Scott G, Parker LS, Graham DI, Doyle D. The contusion index. A quantitative approach to cerebral contusions in head injury. Neuropath Appl Neurobiol 1980;6:319-24.

13 Reilly PL, Graham DI, Adams JH, Jennett B. Patients with head injury who talk and die. Lancet 1975;ii: 375-7.

14 Adams JH. The neuropathology of cerebral hypoxia. In: Camps FE, Robinson AE, Lucas BGB, eds. Gradwohl's Legal Medicine. 3rd ed. Bristol: Wright, 1976;337-48.

15 Brierley JB. Cerebral hypoxia. In: Blackwood W, Corsellis JAN, eds. Greenfield's Neuropathology. 3rd ed. London: Arnold, 1976;43-86.

${ }^{16}$ Graham DI. Pathology of hypoxic brain damage in man. In: Morson BC, ed. Hypoxia and Ischaemia. J Clin Pathol 1977;30 Suppl (Roy Coll Path) 11:170-80.

17 Adams JH, Graham DI The relationship between ventricular fluid pressure and the neuropathology of raised intracranial pressure. Neuropath Appl Neurobiol 1976; 2:323-32.

18 Jennett B, Plum F. Persistent vegetative state after brain damage. Lancet 1972; i:734-7.

${ }^{19}$ Miller D, Adams H. Physiopathology and management of increased intracranial pressure. In: Critchley $\mathbf{M}$, O'Leary JL, Jennett B, eds. Scientific Foundations of Neurology. London: Heinemann, 1972;308-24.

${ }^{20}$ Macpherson P, Graham DI. Correlation between angiographic findings and the ischaemia of head injury. J Neurol Neurosurg Psychiat 1978;41:122-7.

${ }^{21}$ Galbraith S. Misdiagnosis and delayed diagnosis in traumatic intracranial haematoma. $\mathrm{Br} \mathrm{Med} J$ 1976; 1:1438-9.

${ }^{22}$ Teasdale G, Galbraith S. Acute traumatic intracranial haematomas. In: Krayenbuhl $\mathrm{H}$, Maspes PE, Sweet
WH, eds. Progress in Neurological Surgery. Vol. 10. Basel: Karger. In press.

${ }^{23}$ Snoek J, Jennett B, Adams JH, Graham DI, Doyle D. Computerised tomography after recent severe head injury in patients without acute intracranial haematoma. $J$ Neurol Neurosurg Psychiat 1979;42:215-25.

${ }^{24}$ Zimmerman RA, Bilaniuk LT, Gennarelli T. Computed tomography of shearing injuries of the cerebral white matter. Radiology 1978;127:393-6.

${ }^{25}$ Jennett B, Teasdale G, Galbraith S, et al. Severe head injuries in three countries. J Neurol Neurosurg Psychiat 1977;40:291-8.

26 Zimmerman RA, Bilaniuk LT, Bruce D, Dolinskas' C, Obrist W, Kuhl D. Computed tomography of pediatric head trauma: acute general cerebral swelling. Radiology 1978;126:403-8.

27 Kuhl DE, Alavi A, Hoffman EJ, et al. Local cerebral blood volume in head-injured patients. Determination by emission computed tomography of $99 \mathrm{~m} \mathrm{Tc}$-labeled red cells. $J$ Neurosurg 1980;52:309-20.

28 Jennett B, Teasdale G, Braakman R, Minderhoud J, Heiden J, Kurze T. Prognosis of patients with severe head injury. Neurosurgery 1979;4:283-9.

29 Rose J, Valtonen S, Jennett B. Avoidable factors contributing to death after head injury. Br Med J 1977; 2:615-8.

${ }^{30}$ Miller JD, Sweet RC, Narayan R, Becker DP. Early insults to the injured brain. JAMA 1978;240:439-42.

Requests for reprints to: Professor Hume Adams, Institute of Neurological Sciences, Southern General Hospital, Glasgow G51 4TF. 